\title{
Prognostic value of different cardiac MRI parameters for the diagnosis of myocarditis
}

\author{
Waleed Ahmed", Daniel Verdini, Uthamalingam Shanmugam, Heidi S Lumish, Peerawut Deeprasertkul, \\ Yongkasem Vorasettakarnkij, Hector M Medina, Ravi Shah, Thomas J Brady, Udo Hoffmann, \\ Gotdfred Holmvang, Brian Ghoshhajra, David Sosnovik
}

From 2011 SCMR/Euro CMR Joint Scientific Sessions

Nice, France. 3-6 February 2011

\section{Objective}

To evaluate the prognostic utility of different cardiac MRI (CMR) parameters utilized for the diagnosis of myocarditis.

\section{Background}

The established parameters for myocarditis by CMR include $\mathrm{T} 1$ global early enhancement ratio, $\mathrm{T} 2$ ratio and delayed enhancement (DE). Guidelines suggest that two out of three criteria should be positive for diagnosing myocarditis. The prognostic value of these criteria has not been evaluated.

\section{Methods}

Retrospective review of all CMR cases performed for suspected myocarditis over 3 years from 7/2006 to 8/ 2009 was performed. MACE was defined as a composite of cardiac death, persistent NYHA class III or IV, need for ICD and cardiovascular related readmissions during the 1 year after initial presentation.

\section{Results}

209 cases were reviewed; mean age 47 and $58 \%$ men. 74 (35\%) patients were diagnosed with myocarditis based on CMR. The number of patients with $0,1,2$ and 3 criteria positive was $70(33 \%), 65(31 \%), 49(23 \%)$ and 25 (12\%) respectively. Age, gender, diabetes, hypertension, smoking, CAD, prior CHF and CRI were similar among all groups. There was a trend for higher prevalence of NYHA class III or IV in myocarditis patients (17\% vs $7.8 \%$; $\mathrm{P}=0.05)$. Baseline LVEF was $48.44 \pm 18$ by TTE and $46 \pm 21$ by CMR with no significant differences between groups. Mean increase in LVEF at 6 months by
TTE for patients with $0,1,2$ and 3 criteria positive was $5 \pm 10,13 \pm 11,11 \pm 10$ and $6 \pm 12(\mathrm{P}=0.147)$. Mean change in LVEF for three myocarditis groups based on abnormal T1 and T2, T1 and DE or T2 and DE was $10 \pm 9$, $10 \pm 7$ and $7 \pm 9(P=0.39)$. MACE was achieved in 20 (37\%) patients with myocarditis and 21 (23\%) patients without the diagnosis $(\mathrm{P}=0.078)$. The rate of MACE in patients with $0,1,2$ and 3 positive criteria was 11 (22\%), 10 (25\%), 13 (37\%) and 7 (37\%) ( $\mathrm{P}=0.127$ and 0.21 for 0 vs 2 and 0 vs 3 criteria). The three groups based on positive $\mathrm{T} 1$ and $\mathrm{T} 2, \mathrm{~T} 1$ and $\mathrm{DE}$ or $\mathrm{T} 2$ and DE had MACE rates of $3(23 \%), 5(38 \%)$ and $5(38 \%)$ with no significant differences on pairwise comparisons.

\section{Conclusions}

Both the number of criteria positive for myocarditis as well as any particular combination of abnormal parameters were not predictive of improvement in LVEF at 6 months or MACE upto one year. Among patients referred for CMR for suspected myocarditis the outcomes of patients with and without myocarditis by CMR were similar.

Published: 2 February 2011

doi:10.1186/1532-429X-13-S1-P295

Cite this article as: Ahmed et al:: Prognostic value of different cardiac MRI parameters for the diagnosis of myocarditis. Journal of Cardiovascular Magnetic Resonance 2011 13(Suppl 1):P295. 